\title{
A Low-Latency Seismic Recorder for Earthquake Early Warning Chaoyong $\mathrm{PENG}^{1, \mathrm{a}}$, Bing XUE ${ }^{2}$, Jiansi $\mathrm{YANG}^{1}$, Yang $\mathrm{CHEN}^{2}$, Jiang $\mathrm{LI}^{2}$, Minghui LIU ${ }^{2}$, Yinxing $\mathrm{ZHOU}^{2}$ \\ ${ }^{1}$ Institute of Geophysics, China Earthquake Administration, Beijing, 100081, China \\ ${ }^{2}$ Institute of Earthquake Science, China Earthquake Administration, Beijing, 100036, China \\ aemail: pengchaoyong@cea-igp.ac.cn
}

Keywords: Earthquake Early Warning; Seismic Recorder; Low-Latency; EDAS-24GN; Embedded Linux

\begin{abstract}
As one of the effective ways to mitigate earthquake hazards, earthquake early warning (EEW) has been studies by many countries and regions around the world and significant mitigation effectiveness has been achieved during practical application. However, the data format for real-time data transmission in these EEW systems is organized as one packet per second which will lead to certain day when using first three seconds $\mathrm{P}$ wave data for $\tau_{\mathrm{c}} \& \mathrm{P}_{\mathrm{d}}$ calculation. In this case, if $\mathrm{P}$ wave arrival of an earthquake is at the first half of a packet, the data latency will be more than $0.5 \mathrm{~s}$. To address this problem, we develop a new type of seismic recorder called EDAS-24GN with low-latency real-time data output for EEW. It can transfer real-time data by one packet per $0.1 \mathrm{~s}$ so that the leading time can be improved with $0.8 \mathrm{~s}$ at maximum. This will be very useful for areas close to the epicenter.
\end{abstract}

\section{Introduction}

Earthquake early warning system (EEWS) is one of the most effective ways for earthquake disaster mitigation. Many countries and regions around the world have already developed EEWSs, such as California [1][2], Japan [3][4], Mexico [5], Taiwan [6][7], Italy [8], or are developing and testing the prototype systems, including Turkey [9], China [10-13], Iberia [14], etc.

A rapid acquirement of real-time waveform from seismic stations deployed in the epicentral areas is one of the key elements in such system. Therefore, we need to obtain the real-time data with much lower latency. However, the data format of almost all the existent seismic stations are organized as one packet per second for real-time data transmission. In this case, if $\mathrm{P}$ wave arrival of an earthquake is at the first half of a packet, the data latency will be at least $0.5 \mathrm{~s}$ which will lead to shorten the lead time (elapsed time between the alert and damaging wave) and enlarge the blind zone (the radius from the epicenter to the distance traveled by the seismic $S$ wave at the time the alert is issued). In order to address this problem, we have developed a new type of seismic recorder called EDAS-24GN with low latency real-time data output for earthquake early warning (EEW).

\section{Hardware Structure}

The hardware structure of EDAS-24GN is shown in Figure 1. It consists of a data acquisition unit, a sensor control module, a calibration signal generation unit, a GPS antenna, a FPGA logic control, an AT91SAM9263 CPU with $200 \mathrm{MHz}$, and various types of peripherals and storage devices. As shown in Figure 1, the hardware structure of the new type of seismic recorder is very similar to those of traditional ones [15]. The only difference is that this recorder has no DSP and all the real-data filtering operation (FIR) is performed on CPU. 


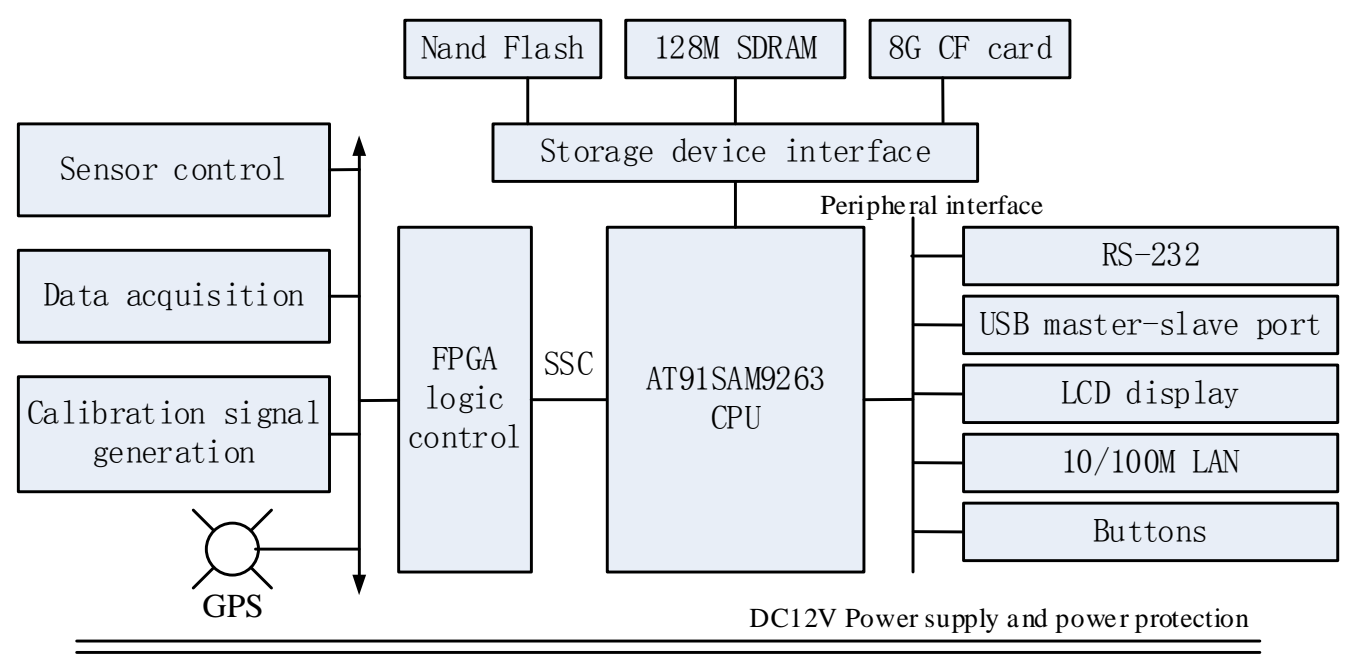

Fig.1. Hardware structure of EDAS-24GN

\section{Low-Latency Data Processing}

To acquire data of ground shaking as soon as possible for estimating seismic parameters such as magnitude, focal depth and epicentral distance according to the initial vibration of P-wave [16-18] and determining extent and level of EEW, we changed the mode of data organization from one packet per second to the format with a real-time data stream buffer and a time-index pointer list (Figure 2).

For each channel and sampling rate, there are a real-time data buffer and a corresponding time-index pointer list which can cache data of $20 \mathrm{~s}$. When a user starts fetching real-time data, he needs to set sampling rate, phase type and data frame length (fra_len). The system will first locate position of the latest second (newest_sec) according to sampling rate and phase type. Then real-time data will be fetched with the length defined by fra_len. If the data length between last acquired data position (last_pos) and the latest data point (newest_data) is more than fra_len, the real-time data will be continuously fetched and last_pos will be updated as last_pos plus fra_len until the length of the rest data is smaller than fra_len. This will ensure that length of the delayed points is less than fra_len. If fra_len and sampling rate are set as 20 and $200 \mathrm{~Hz}$ respectively, the real-time data latency will be less than $0.1 \mathrm{~s}$.

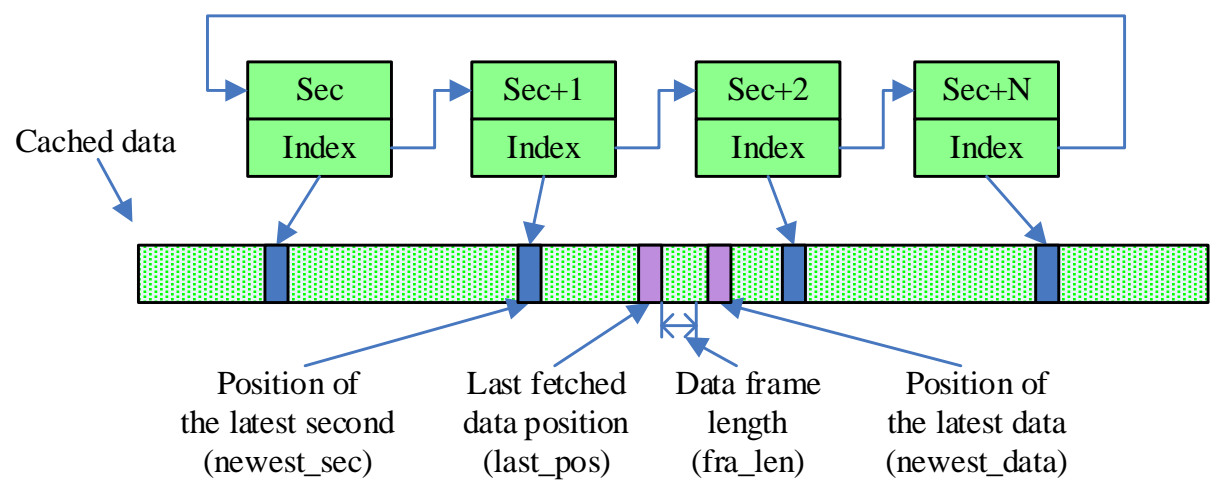

Fig.2. Low-latency data organization

\section{Real-Time Data Compression}

Generally the time interval of $0.1 \mathrm{~s}$ is set as the output speed of data frame for low-latency real-time data transmission. In this case, for a three-component seismic recorder with sampling rate of $100 \mathrm{~Hz}$, each real-time data frame has only 30 sampling points. Due to the small amount of each frame, length of additional information such as waveform information and coding information must be reduced in order to shorten the overall frame length of compressed data. 
For this reason, we designed a new data compression algorithm using a more refined delta encoding method for low-latency data transmission. There are two key steps in the method. One is further block encoding for differences between sequential data in order to avoid increase of frame length existed in STEIM2 algorithm due to sudden changes of individual sample values in the real-time data series. The other one is to create a two-dimensional coding table through statistical analysis of differences between waveform data. Then we can determine the actual code length by looking up the table when compressing and decompressing data. The compressed data frames only contain index information of the table, and its length is only related to length of the original waveform data.

The comparison between results of the new method and those of the STEIM2 algorithm is shown in Table 1. For the STEIMS algorithm, if the code length is more than 20 bits, the final compressed data length will be extended and longer than the original data length. The reason is that only one code with 31 bits is defined for data with more than 15 effective bits, and more coding description fields are needed to describe encoding length of differences in the data frame. The new method doesn't extend length of compressed data and its compression ratio is better than that of the STEIMS algorithm.

Table 1. Comparison between results of the new method and those of the STEIM2 algorithm

\begin{tabular}{cccccc}
\hline \multirow{2}{*}{$\begin{array}{c}\text { Code } \\
\text { length }\end{array}$} & $\begin{array}{c}\text { Original } \\
\text { length }\end{array}$ & \multicolumn{2}{c}{ New algorithm } & \multicolumn{2}{c}{ STEIM2 algorithm } \\
\cline { 3 - 6 } (bit) & $\begin{array}{c}\text { Compressed } \\
\text { length }\end{array}$ & $\begin{array}{c}\text { Compression } \\
\text { ratio }\end{array}$ & $\begin{array}{c}\text { Compressed } \\
\text { length }\end{array}$ & $\begin{array}{c}\text { Compression } \\
\text { ratio }\end{array}$ \\
\hline 5 & 560 & 105 & 5.33 & 126 & 4.44 \\
15 & 560 & 276 & 2.03 & 322 & 1.74 \\
20 & 560 & 344 & 1.63 & 602 & 0.93 \\
29 & 560 & 480 & 1.17 & 602 & 0.93 \\
\hline
\end{tabular}

\section{Discussion and Conclusion}

The purpose of EEWS is to get more than enough lead-time for disaster mitigation. However, almost all the existent seismic stations used for EEWS transfer real-time data with the format of one packet per second, which will lead to certain data latency when using $3 \mathrm{~s}$ of $\mathrm{P}$ wave data for $\tau_{\mathrm{c}} \& \mathrm{P}_{\mathrm{d}}$ calculation. Especially when the $\mathrm{P}$ wave arrival of an earthquake is at the first half of a packet, the data latency will be more than $0.5 \mathrm{~s}$.

As shown in Figure 3(a), assuming that $P$ wave arrives at $0.1 \mathrm{~s}$ and $S$ wave arrives at $3.9 \mathrm{~s}$, if we use the data organization of existent EEWSs, the initial $\mathrm{P}$ wave data will be obtained at $1 \mathrm{~s}$ and early-warning parameters will be calculated at $4 \mathrm{~s}$. In this case, there will be data latency of $0.9 \mathrm{~s}$ and no warning will be released for the simulated event.

(a)

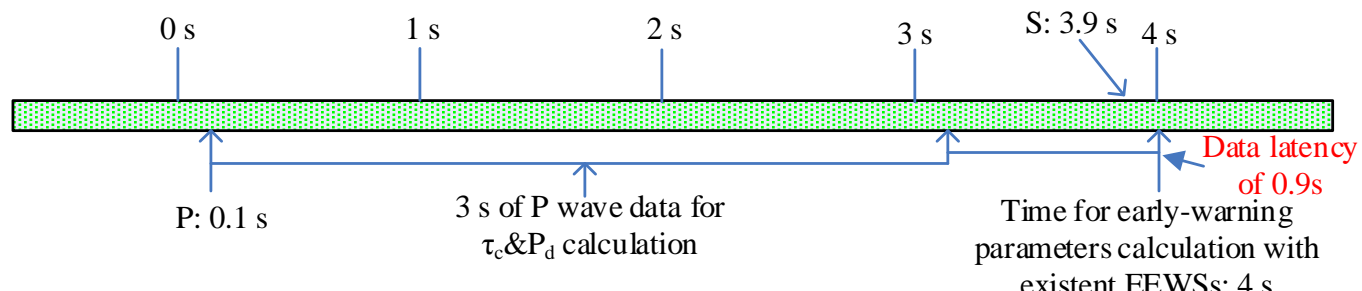

(b)

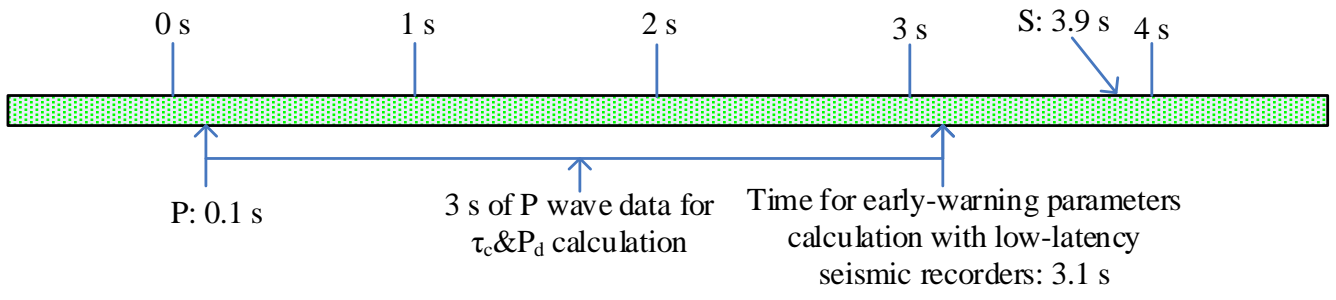

Fig.3. Comparison of $\tau_{\mathrm{c}} \& \mathrm{P}_{\mathrm{d}}$ calculating time of existent and low-latency EEWSs (a) $\tau_{\mathrm{c}} \& \mathrm{P}_{\mathrm{d}}$ calculating time of existent EEWSs; (b) $\tau_{\mathrm{c}} \& \mathrm{P}_{\mathrm{d}}$ calculating time of low-latency EEWS 
However, if we adopt the data organization of the new developed low-latency seismic recorder, effective lead time can be provided for the simulated event, shown in Figure 3(b). The initial $\mathrm{P}$ wave data can be obtained at $0.1 \mathrm{~s}$ with transmission interval of $0.1 \mathrm{~s}$. Then the whole $3 \mathrm{~s}$ of $\mathrm{P}$ wave data using for early-warning parameters calculation will be completely collected at $3.1 \mathrm{~s}$. Therefore, effective lead time of $0.8 \mathrm{~s}$ will be acquired for this case.

From the above analysis, the effective lead time improved by this new seismic recorder is closely related to $\mathrm{P}$ wave arrival. If $\mathrm{P}$ wave arrival is at the beginning of $1 \mathrm{~s}$ data, the effective lead time can be increased at least $0.5 \mathrm{~s}$. Otherwise if $\mathrm{P}$ wave arrival is at the end of $1 \mathrm{~s}$ data, the improved effective lead time will be very limited, such as $0.1 \mathrm{~s}$.

Assuming that $\mathrm{S}$ wave velocity is $3 \mathrm{~km} / \mathrm{s}$, EEWS installing low-latency seismic recorders can reduce the blind zone with $0.3 \mathrm{~km}(0.1 \mathrm{~s})$ to $2.7 \mathrm{~km}(0.9 \mathrm{~s})$. If the blind zone of existent EEWSs is $30 \mathrm{~km}$, it can be reduced to $27.3 \mathrm{~km}$ at most with low-latency seismic recorders. Because the increased lead time using low-latency seismic recorders doesn't vary with external conditions, in this case, the closer to the epicentral area, the more advantages of the new EEWS can be demonstrated. As can be seen from Figure 3, although lead time of only $0.8 \mathrm{~s}$ is obtained for the simulated earthquake, the time is sufficient for us to take appropriately automatic emergency actions for some important infrastructures, such as slowing down high-speed trains and shutting down nuclear facilities, in order to further reduce losses caused by earthquakes. The low-latency seismic recorders have been deployed in large quantities to the prototype EEWS developed by Fujian Provincial Seismological Bureau [11].

\section{Acknowledgement}

This work was financially supported by Basic Research Project of Institute of Geophysics, China Earthquake Administration (DQJB14B05) and the National Natural Science Foundation of China (41404048).

\section{References}

[1] Richard M. Allen, Hiroo Kanamori. The potential for earthquake early warning in southern California [J]. Science, 2003300 786-789.

[2] Richard M. Allen, Holly Brown, Margaret Hellweg, Alexei Kireev, Douglas Neuhauser. Real-time test of the ElarmS earthquake early warning methodology [J]. Journal of Geophysical Research, 2007112 B08311.

[3] Osamu Kamigaichi, Makoto Saito, Keiji Doi, Toshiyuki Matsumori, Shin’ya Tsukada, Kiyoshi Takeda, Toshihiro Shimoyama, Kouji Nakamura, Masashi Kiyomoto, Yukihiro Watanabe. Earthquake early warning in Japan: Warning the general public and future prospects [J]. Seismological Research Letters, 2009 80(5) 717-726.

[4] Yutaka Nakamura. UrEDAS, Urgent earthquake detection and alarm system, now and future [C]. Proceeding of $13^{\text {th }}$ World Conference on Earthquake Engineering, 2004.

[5] A. Iglesias, S.K. Singh, M. Ordaz, M.A. Santoyo, J. Pacheco. The seismic alert system for Mexico City: An evaluation of its performance and a strategy for its improvement [J]. Bulletin of the Seismological Society of America, 2005 97(5) 1718-1729.

[6] Yih-Min Wu, Hiroo Kanamori. Development of an earthquake warning system using real-time strong motion signals [J]. Sensors, 2008 8(1) 1-9.

[7] Nai-Chi Hsiao, Yih-Min Wu, Tzay-Chyn Shin, Li Zhao, Ta-Liang Teng. Development of an earthquake early warning system in Taiwan [J]. Geophysical Research Letters, 200936 L00B02.

[8] G. Iannaccone, A. Zollo, L. Elia, V. Convertito, C. Satriano, C. Martino, G. Festa, M. Lancieri, A. Bobbio, T.A. Stabile, M. Vassallo, A. Emolo. A prototype system for earthquake early-warning 
and alert management in southern Italy [J]. Bulletin of Earthquake Engineering, 2010 8(5) 1105-1129.

[9] M. Erdik, Y. Fahjan, O. Ozel, H. Alcik, A. Mert, M. Gul. Istanbul earthquake rapid response and the early warning system. Bulletin of Earthquake Engineering, 2003 1(1) 157-163.

[10] Hanshu Peng, Zhongliang Wu, Yih-Min Wu, Shuming Yu, Dongning Zhang, Wenhui Huang. Developing a prototype earthquake early warning system in the Beijing Capital Region [J]. Seismological Research Letters, 2011 82(3) 394-403.

[11] Xing Jin, Yongxiang Wei, Jun Li, Hongcai Zhang, Qiang Ma, Lanchi Kang. Progress of the earthquake early warning system in Fujian, China [J]. Earthquake Science, 2013 26(1) 3-14.

[12] Chaoyong Peng, Jiansi Yang, Bing Xue, Yang Chen, Xiaoyi Zhu. Research on correlation between early-warning parameters and magnitude for the Wenchuan Earthquake and its aftershocks [J]. Chinese Journal of Geophysics - Chinese Edition, 2013 56(10) 3404-3415.

[13] Chaoyong Peng, Xiaoyi Zhu, Jiansi Yang, Bing Xue, Yang Chen. Development of an integrated onsite earthquake early warning system and test deployment in Zhaotong, China [J]. Computers \& Geosciences, 201356 170-177.

[14] M. Picozzi, S. Colombelli, A. Zollo, M. Carranza, E. Buforn. A threshold-based earthquake early-warning system for offshore events in Southern Iberia. Pure and Applied Geophysics, 2015, doi:10.1007/s00024-014-1009-2.

[15] Hongti Wang, Cantao Zhuang, Bing Xue, Jiang Li, Yang Chen, Xiaoyi Zhu, Wenyu Lou, Minghui Liu. A network communication and recording system for digital seismic observation [J]. Acta Seismologica Sinica, 2006 28(5) 540-545.

[16] Andrew B. Lockman, Richard M. Allen. Single-station earthquake characterization for early warning [J]. Bulletin of the Seismological Society of America, 2005 95(6) 2029-2039.

[17] Yih-Min Wu, Li Zhao. Magnitude estimation using the first three seconds P-wave amplitude in earthquake early warning [J]. Geophysical Research Letters, 200633 L16312.

[18] Yih-Min Wu, Hiroo Kanamori, Richard M. Allen, Egill Hauksson. Determination of earthquake early warning parameters, $\tau_{c}$ and $P_{d}$, for southern California [J]. Geophysical Journal International, 2007170 711-717. 